\title{
FUTURE OF THE FACE: THE PHOTOGRAPH AND THE ALGORITHM
}

\author{
Elisabeth Stoney \\ Asst. Prof. Dr, Zayed University, UNITED ARAB EMIRATES, elisabethstoney@gmail.com
}

\begin{abstract}
Photography was pronounced dead in the 1980 s following the widespread introduction of digital cameras. At the time it was considered among photographic historians that the innovation of the digital pixel, that element that allowed for endless cloning and manipulation of the image, was the defining factor of the new photographic technology. My paper argues that digital photography, in its radical difference from the technologies of chemical-based photography, needs to be theorised within the context and the development of digital information, from where the role of the digital photograph within innovative computer-aided imagery can be critically considered. I argue that our understanding of what photography is and what it is yet to become, needs to recognize the direction of informational technologies to which photography is ever more connected.

This paper considers contemporary advances in a range of computer-aided imaging. There are attendant shifts in our daily uses and practices of photography that operate on increasingly personalised and diaristic planes. But while the 'selfie', tagging and facial recognition are the everyday realities of social media usage and the online traffic in images proliferates and accelerates, developments in computational photography such as digital biometrics, recognition, stitching and 3D reconstruction, remain lesser known and expert fields at the forefront of informational technology destined for use in security systems, market research and intelligence.

Mark Zuckerberg's prediction that 'the future will be social' is also a confirmation that the future will also be networked, that the digital interaction of users and experiences, call it 'big data', relies on a connected online world of users. In this post-photographic moment, the photograph still exists but in series, associations, connections and archives. Also the photograph is social. The single photograph, captured in a decisive moment, is of the past. In this paper I consider recent advances in the field of image technology to analyse the directions in which the traffic in photography is flowing.
\end{abstract}

Keywords: photography, photographic history, algorithm, information technology, digital, face recognition, biometrics, interconnectivity, 3D construction, computer-aided imagery.

\section{INTRODUCTION}

Since 1988, when Fuji marketed the first digital camera for consumer use, theorists have written extensively on the impact of digital technology in the shift from the chemical to the digital photograph. At its 150th year 
anniversary in 1989, photography was pronounced 'dead', or at least 'radically and permanently displaced' (Mitchell, 20). The introduction of digital photography marked a radical shift in how photographs were produced at a technical level, while, in the ways photographs were shot, stored and shared, our habits and practices began to change, eventually with an increased engagement with the web. In effect, we transformed the role and meaning of photography in our lives.

At the time, the digital pixel was identified as the key element, the innovation that allowed for the limitless cloning and manipulation of the image and the defining factor of the new photographic technology. Debates around the photograph's relationship to the real as a physical index 'adherent to the referent' began to come apart, while questions of the agency and originality of the photographer/auteur came under radical revision (Mitchell). Even so, out of these ruins emerged an optimism for the potential of photography where the creation and distribution of the digital image was in the hands of so many. In 2007 the Musée de l'Elysée of Lausanne launched an exhibition entitled We Are All Photographers Now! - an interactive event that celebrated the abolition of the distinctions between the professional and amateur, fine print photography and the vernacular. And yet if everyone is a photographer - what exactly is a photographer? How then does photography implicate us all?

\section{THE DEMATERIALISATION OF THE PHOTOGRAPH}

The control (or lack of it) and the ownership and rights over the digital image have remained constant legal and critical concerns. The camera as an instrument, as a stand-alone differentiated device, merged inexorably first with the personal computer and then with the telephone. The $\mathrm{pc}$ and the telephone are the everyday user interfaces of network-based computing, since 2006 integrated with cloud technology for expanded data storage for individuals and commercial enterprises. If you have accessed social media then you have accessed the cloud, a network of remote servers hosted on the Internet to store and process data. IPhone 6 is promoted by Apple foremost as a digital camera on the basis of the image quality of the camera function. Nevertheless at the level of technology and function, the mobile telephone functions more accurately as a terminal, the interface that connects the user to the mainframe.

The single static photograph, a fragment isolated and un-plugged, has now little identity. At best it is latent heritage that requires research and conservation. Moreover it is the data-mapping and the relationships developed between data that produce meaning. As post-photographic, the photograph exists only through activity, in its proliferation or association. So the photograph is as social as the user. The single photograph, captured in a decisive moment, is invisible, until it can be reimagined as a type, or one of a series or archive. All analogue images belong to a silent, invisible archive that awaits revival via a curator or a scanner. Google vice president and internet pioneer Vint Cerf suggests nevertheless that a similar fate or worse may be awaiting our digital memories, warning that the 21 st century could become a second 'Dark Ages' if our aging files become technologically inaccessible. His advice is to print out treasured photographs (Knapton, 2015).

To discuss the photograph as a thing, an object, barely makes sense today. While we arguably print more photographs today than ever, and while over two billion photographs are uploaded daily, a photograph is no longer a print, nor exactly a screen image. If we no longer associate the photograph with print media it is because photographs are more likely to pass fleetingly in our lives across an accelerating number of competing screens where the screen platform seems to host the ephemerality of the digital image in its emergence and disappearance rather than defining the photograph anew. Like a new medium, like an encounter with a new silent cinema, the photograph, now 'radically displaced', transforms itself. Images flow in communion with others, emerging and submerging only to reappear in other moments and other sites. The digital image/photograph is an ethereal presence that exists to co-exist, recalling Michel Serres's quasiobject - the object 'in the chain and of the chain', between being and relating, its work being to identify the subject who is dependent on it (Serres). The digital photograph, like the quasi-object, is an activity and a communication, a relational tool where 'the virtual creates the real,' even a community (Obrist, 121). Recent accounts of the history of photography, such as Kaja Silverman's The Miracle of Analogy that addresses photography of the pre-digital era, steer away from material-based and medium-specific understandings of the medium. Silverman also locates photography as relational, as a philosophical thread between the human being and the world - that world requesting of the human a response to its beauty. For Silverman, photography is that response (Silverman).

\section{NEW PHOTOGRAPHIC TECHNOLOGIES}

Evidently the uses of photography are not however limited to the glorification of the universe. In the light of the information in the public domain since 2013 on the monitoring conducted by the National Security Agency (NSA), an intelligence organization of the US government, the cloud, which had been imagined as a 
safe and almost heavenly storage location, became a focus of disenchantment with digital forms on the part of some users. According to the documents revealed by IT engineer Edward Snowden, global technology giants (including Microsoft, Yahoo, Facebook and Google) had agreements with the NSA whereby the agency illegally acquired access to an enormous scope of personal and confidential data for use in surveillance (Ackerman \& Rushe).

When Tung-Hui Hu, author of $A$ Prehistory of the Cloud, notes that 'you're all sharing the same computer and yet you think of this as private' he refers to both the communality of the internet and also its liabilities (Sutcliffe). The cloud is a system of energy-hungry data servers that can function as a crowd-sourcing mechanism for data to be accessed by those able to afford it. It is both material and virtual. 'Much more than a data center,' Lisa Parks argues, 'the cloud is a diffuse and invisible structure of power that has yielded a data-centric order' (https://mitpress.mit.edu/prehistory-cloud). The most glaring example of this crowdsourcing of data is the 'selfie' and the associated use of tagging, where internet users collect and categorize personal and biometric data, all voluntarily uploaded to social media applications. And yet social media applications and the store of faces are the tip of the iceberg for recognition algorithms. Facebook's artificial intelligence lab is developing algorithms that recognize individuals from other physical attributes such as clothing, body-shape and pose, all legible even when faces are not visible (Rutkin). With 200 images of an individual taken randomly from the internet or elsewhere, it is possible to simulate his/her speech - the algorithm can also be adapted to transpose words into the mouths of others. Obama can appear to speak like Bush.

An analysis of developments in computer-aided technology suggests that the range of new software applications working with photographic images are being developed to process and elaborate information from big data. Much computer-aided software, such as stitching, 3D construction and texture mapping, works from photographic images to simulate 3D environments and virtual realities. One controversial example, which is since 2015 in its second developmental stage after the critical failure of the original version, is Google Glass, a networked optical device, worn as a pair of glasses and designed to capture, record and interact with visual data (Crothers). For Wikileaks founder Julian Assange, the placement of Google's device between one's eyes and the world is a totalitarian concept and 'a realization of Orwellian horror' (Rattansi). The design and the use of certain recent photographic technologies do appear to be designed to render the world as a unified image in three or more dimensions, in a personalized adaption of Google Earth. Importantly however, Google Glass and its competitor product being developed by Dispelix Oy, appear to return the human body in its dynamism, its eye and brain, to the centre of image-making and meaning.

Mark Zuckerberg made a series of predictions in 2015 regarding the future of augmented reality, which he believes will revolutionize our internet use and become the most important marketing platform over the next ten years (Del Prado). He made further predictions including the introduction of laser-beamed wifi and telepathic communication, as well as intensification in the role of the sms and the photograph in messaging. Neither snapping nor chatting will fade away, it seems, but rather dominate the models of social media of tomorrow.

\section{REFERENCE LIST}

Ackerman, S. \& Rushe, D. (2014). Microsoft, Facebook, Google and Yahoo release US surveillance requests. The Guardian, 3 February. http://www.theguardian.com/world/2014/feb/03/microsoftfacebook-google-yahoo-fisa-surveillance-requests.

Crothers, B. (2015). The Google Glass Epic Fail: What Happened? BGR 27 June, http://bgr.com/2015/06/27/google-glass-epic-fail-what-happened/.

Del Prado, G.M. (2015). Mark Zuckerberg's vision of the future is full of artificial intelligence, telepathy, and virtual reality. Businessinsider, 2 July, http://www.businessinsider.com/facebooks-mark-zuckerbergpredictions-about-the-future-2015-7.

Hu, T-U. (2015). A Prehistory of the Cloud. Cambridge Massachusetts: MIT Press.

Knapton, S. (2015). Print out digital photos or risk losing them, Google boss warns. The Telegraph, 13 February. http://www.telegraph.co.uk/news/science/science-news/11410506/Print-out-digital-photosor-risk-losing-them-Google-boss-warns.html.

Mitchell, W. (1994). The Reconfigured Eye - Visual Truth in the Post-Photographic Era. Massachusetts: MIT Press.

Obrist, H. U. (2013-2014). Michel Serres, 02C, vol. 25. 
Rattansi, A. (2014) Julian Assange: 'Orwellian horror' of Google Glass. GoingunderRT. https://www.youtube.com/watch?v=OTV_Vz-Ur2M.

Rutkin, A (2015). Facebook can recognise you in photos even if you're not looking. New Scientist, 22 June.

Serres, M. (1982). The Parasite. Trans. Lawrence R. Schehr. Baltimore: Johns Hopkins University Press.

Silverman, K. (2015). The Miracle of Analogy, or The History of Photography, Part I. Stanford, CA: Stanford University Press.

Sutcliffe, J. (2015). A Prehistory of The Cloud: an interview with Tung-Hui Hu. Rhizome, 16 December. http://rhizome.org/editorial/2015/dec/16/interview-tung-hui-hu/.

(2015). A Prehistory of the Cloud - Endorsements. https://mitpress.mit.edu/prehistory-cloud. 